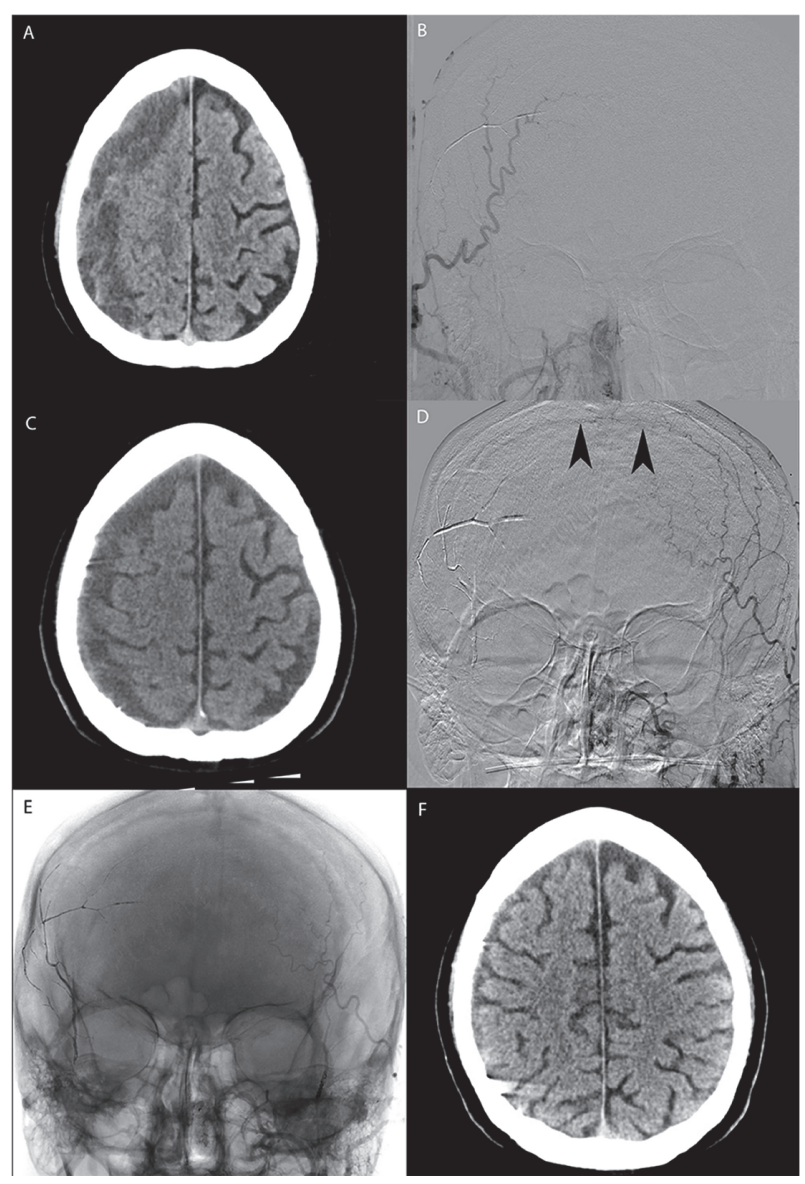

Abstract E-111 Figure 1

underwent contralateral MMAE. Both patients were neurologically intact with radiographic improvement of $\mathrm{CSDH}$ at follow up.

Conclusion Re-accumulation of SDH following MMAE by means of contralateral MMA neovascularization is a rare subtype of SDH recurrence following MMAE. Within the context of re-accumulation of SDH following MMAE catheter angiography is an important diagnostic investigation to elucidate the etiology of the recurrence. Furthermore, when angiography reveals neovascularization of the contralateral MMA, embolization of the contralateral MMA achieves good clinical and radiographic result.

Disclosures Z. Hubbard: None. S. Al Kasab: None. G. Porto: None. M. Sattur: None. A. Spiotta: None.

\section{E-112 INFANTILE TRAUMATIC PERICALLOSAL ANEURYSM: A CASE REPORT}

Z Hubbard*, G Porto, S Al Kasab, M Sattur, A Spiotta. Medical University of South Carolina, Charleston, SC

\subsection{6/neurintsurg-2021-SNIS.207}

Background and Importance Traumatic aneurysms are a rare sequelae of non-accidental head trauma in infants. The rate of non-accidental trauma in the pediatric population is increasing, therefore traumatic aneurysms are an important consideration in the evaluation of pediatric patients with abusive head trauma. We report a 4-week-old infant who suffered a traumatic aneurysm arising from the pericallosal artery.

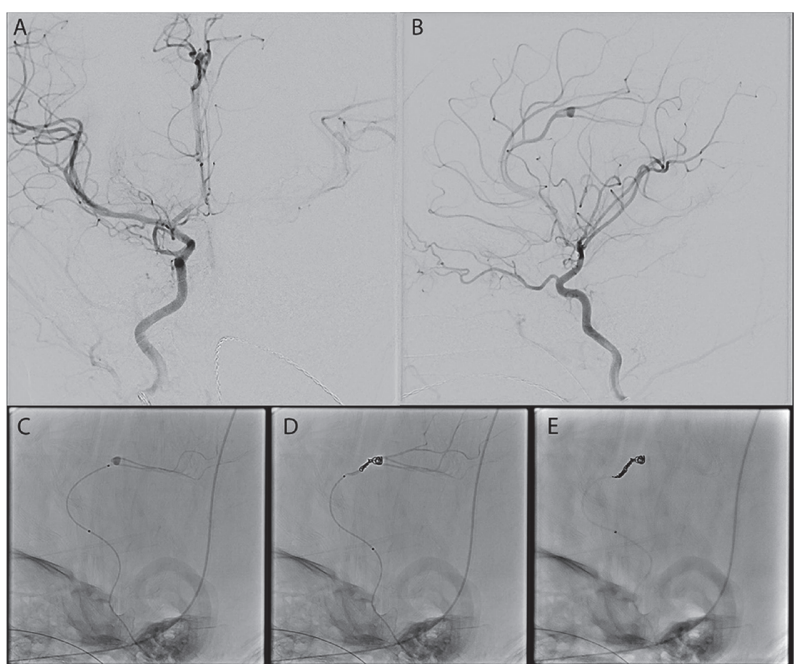

Abstract E-112 Figure 1

Case Presentation A 24 day old infant with no significant past medical or birth history presented with twitching and poor oral intake for one day prior to presentation. The patient was found to have bilateral SDH, multifocal contusions, and traumatic subarachnoid hemorrhage. NAT workup was remarkable for repeated and prolonged period of abuse. MRA revealed a pericallosal traumatic aneurysm that was treated by means of onyx/coil embolization.

Conclusion Traumatic intracranial aneurysms are a rare, but serious sequelae of pediatric abusive trauma. The authors present a case of a pericallosal traumatic aneurysm as a result of abuse that was treated with coil/onyx embolization of the aneurysm and parent artery occlusion. Rates of pediatric abuse are increasing, and as such traumatic intracranial aneurysms will remain a challenging entity to identify and treat. Despite new methods for management of traumatic aneurysms, prognosis remains poor due to the diffuse injury often involved in these patients.

Disclosures Z. Hubbard: None. G. Porto: None. S. Al Kasab: None. M. Sattur: None. A. Spiotta: None.

\section{E-113 ENDOVASCULAR RECURRENCE OF CIRCLE OF WILLIS ANEURYSMS: RE-ACCESS FOLLOWING FLOW DIVERSION}

${ }^{1} \mathrm{~J}$ Steele* ${ }^{2}{ }^{\mathrm{T}}$ Payner, ${ }^{2} \mathrm{C}$ Kulwin, ${ }^{2} \mathrm{D}$ Sahlein. ${ }^{1}$ Michigan Head and Spine Institute, southfield, MI: ${ }^{2}$ Goodman Campbell Brain and Spine, Carmel, IN

\subsection{6/neurintsurg-2021-SNIS.208}

Introduction Clinically significant aneurysm recurrence after surgical clipping, regardless of size, is low at 1\%. Clip reconstruction of large and giant aneurysms, however, have a higher rate of retreatment of $8 \%$ and consequent patient morbidity (12\%) and mortality (7\%). Endovascular flow diversion has decreased the treatment morbidity and mortality $(7 \%, 4 \%$ respectively) of large and giant aneurysms significantly with 5 year residual aneurysm rate of $5 \%$. Further flow diversion of residual aneurysm regrowth has the potential for occluding parent vessel branches and increasing patient morbidity. The authors present a case series of endovascular aneurysm recurrence following flow diversion treated by using the Circle of Willis to directly access and treat the aneurysm recurrence from the adjacent circulation. 